\title{
La madurez ante el espejo: Interrelación entre la satisfacción corporal y vital de personas mayores en España
}

\author{
Roberto Sánchez Cabrero, Amelia Barrientos Fernández y \\ Amaya Arigita García \\ Universidad de Nebrija (España)
}

\begin{abstract}
La satisfacción corporal y vital está íntimamente relacionadas durante la juventud y edad adulta. No obstante, en la vejez ambos procesos padecen severas transformaciones de forma aislada que podrían modificar la naturaleza de esta relación significativamente. Este estudio se plantea evaluar si la relación entre satisfacción corporal y vejez se mantiene estable durante la vejez y cuáles son los factores que determinan la naturaleza de dicha relación, valorando la edad, el sexo, la presencia de pareja y el entorno como variables de estudio. Se utiliza el Body Shape Questionnaire (BSQ. Cooper, Taylor, Cooper, y Fairburn, 1987, adaptado y validado sobre población española por Raich et al., 1996) para medir la satisfacción corporal, y el Cuestionario de satisfacción Vital (CSV. Sánchez, 2012) para medir la satisfacción vital. Los resultados indican que la mayor insatisfacción corporal a partir de los 65 años se asocia directamente con ser mujer, habitar en un entorno urbano y tener pareja actual. A partir de los 50 años la satisfacción corporal y vital sigue correlacionando fuertemente para las mujeres, pero las diferencias observadas según sexo están mediatizadas por la presencia de pareja, que tiene un funcionamiento inverso para hombres y mujeres, y enmascara la posible influencia del factor sexo en los participantes al estudio.
\end{abstract}

Palabras Clave: Satisfacción corporal, satisfacción vital, imagen corporal, madurez, vejez.

The maturity before the mirror: Interrelation between the corporal and vital satisfaction of the elderly in Spain. Body and life satisfaction are intimately related during youth and adulthood. However, in old age both processes suffer severe transformations that could significantly modify the nature of this relationship. This study aims to assess whether the relationship between body satisfaction and old age remains stable during old age and what are the factors that determine the nature of this relationship, assessing age, sex, presence of a couple and the environment as study variables. It is used the Body Shape Questionnaire (BSQ. Cooper, Taylor, Cooper, \& Fairburn, 1987, adapted and validated on Spanish population by Raich et al., 1996) to measure body satisfaction, and the Vital Satisfaction Questionnaire (CSV. Sánchez, 2012) to measure life satisfaction. The results indicate that the greatest body dissatisfaction after 65 years of age is directly associated with being a woman, living in an urban environment and having a current partner. After 50 years, body and vital satisfaction continues to correlate strongly for women, but the differences observed according to sex are mediated by the presence of a couple, which has an inverse functioning for men and women, and masks the possible influence of the sex factor in the study participants.

Keywords: Body satisfaction, life satisfaction, body image, maturity, old age.

Correspondencia: Roberto Sánchez Cabrero. Facultad de Educación. Universidad de Nebrija. C/ Santa Cruz de Marcenado, nº 27. C.P.: 28015. Madrid (España). E-mail: rsanchezca@ nebrija.es 
Se puede definir conceptualmente a la imagen corporal, tomando como referencia autores de gran relevancia en el campo como Thompson (2004), Rosen (2006) o Cash (2017), como la manera en que uno percibe, imagina, siente y actúa respecto a su propio cuerpo. La imagen corporal está formada por dos dimensiones básicas, la dimensión perceptiva, estimación del tamaño y proporciones del propio cuerpo, y la dimensión cognitivo-emocional, también denominada satisfacción corporal, y que va a ser la variable utilizada en este estudio (Cash, 2017). En personas en proceso de madurez y vejez los problemas relacionados con una imagen corporal negativa suelen basarse exclusivamente en el componente cognitivo-emocional de la imagen corporal (satisfacción corporal), ya que la distorsión perceptiva del tamaño o proporciones del cuerpo propio, común en los trastornos de la imagen corporal durante la adolescencia y juventud, no suele darse a estas edades (Davison y McCabe, 2005; Karazsia, Murnen, y Tylka, 2017; Kozar, 2005; Sabik y Bersey, 2016).

El principal motivo por el cual la distorsión perceptiva propia de los trastornos asociados a la imagen corporal en la adolescencia o juventud no suele darse en personas maduras o mayores es el cambio en el foco de preocupación corporal. Está demostrado que el tema central de la preocupación por la apariencia física en personas maduras o mayores son los signos de la edad, es decir, arrugas, caída de pelo, pérdida de condiciones físicas, olor corporal, etc. (Gubrium y Holstein, 2006; Zeman y Zeman, 2015), a diferencia de otras edades, en las que la delgadez es la principal preocupación sobre la apariencia (Maganto, Garaigordobil, y Kortabarria, 2017; Raich, 2004; Rosen, 2006). El carácter evolutivo de los signos de la edad favorece la aceptación de la persona de su propio cuerpo, aunque también favorece una menor satisfacción corporal (al alejarse más del ideal deseado) y una visión negativa del futuro en relación a su aspecto físico. Estas diferencias justifican que en la madurez se den menos casos de trastornos alimentarios y más casos de depresión asociados a la denominada "crisis de madurez", que se desencadena a través del reconocimiento en uno mismo de los signos de la edad propios de la vejez (Gubrium y Holstein, 2006; Hudson, Lucas, y Donnellan, 2016).

La escasez de casos de trastornos alimentarios en la madurez y la vejez, y el cambio en el tema central de la preocupación por la apariencia física han provocado que se generen muy pocas investigaciones que estudien la imagen corporal en este periodo de edad. Según la base de datos informatizada de la APA (PsycNet, 2018), en los últimos cinco años las investigaciones que estudian la imagen corporal desde la madurez o la vejez no llegan al $5 \%$ del total.

En el presente estudio se ha establecido la edad de 50 años como punto de corte para el inicio de la madurez personal, por ser la edad más aceptada por los estudios recientes (Mellor, Connaughton, McCabe, y Tatangelo, 2017; Sánchez y Maganto, 2018) y la edad de 65 años se ha establecido como inicio de la vejez, ya que es la edad a la que 
se produce la jubilación laboral obligatoria en España y conlleva un punto de inflexión importante.

La jubilación laboral se asocia socialmente directamente con el inicio de la vejez, ya que las personas suelen identificar vejez con retiro, descanso y fin de la productividad laboral (Vega, Ramos, Barrios, y Quintero, 2015).

El sexo de la persona sigue siendo un factor de gran influencia sobre la satisfacción corporal a partir de los 50 años, tal y como atestiguan estudios de actualidad (Homan y Tylka, 2018; Murray y Lewis, 2014; Sánchez y Maganto, 2018) en los que se pone en evidencia que ser mujer se asocia directamente a una menor satisfacción corporal también a partir de esta edad.

Otro factor que muestra gran relevancia científica sobre la satisfacción corporal a partir de los 50 años es la presencia o ausencia de pareja. Numerosos estudios muestran evidencias claras de que ambos factores están íntimamente relacionados de forma directa (Aberg et. al., 2006; Boyes, Fletcher, y Lattner, 2007; Davidson y McCabe, 2005) aunque estudios como el de Markey, Markey, y Birch (2001) afirman que la satisfacción corporal de las mujeres sólo está directamente relacionada con la idea distorsionada de la opinión de su pareja sobre su aspecto físico.

El avance de las tecnologías de la comunicación a distancia y de las comunicaciones entre localidades alejadas de las grandes urbes ponen en duda la influencia actual de un factor tradicionalmente de gran relevancia para la satisfacción corporal como lo es el hábitat de convivencia, urbano o rural (Cash, 2017; Sánchez, 2012). Es de gran interés valorar hasta qué punto la influencia de este factor sobre la satisfacción corporal se presenta en las localidades más rurales.

En relación a la otra variable dependiente evaluada en el estudio, se define la satisfacción vital como la impresión personal sobre las condiciones actuales en las que se desarrolla la vida propia. En ésta se engloban distintos aspectos de la persona como salud, red social o condiciones económicas, a través del filtro que supone la personalidad, historia vital, estado anímico y autoconcepto personal del propio sujeto(Chacón-Borrego, Corral-Pernía y Castañeda-Vázquez, 2017; Parra-Rizo, 2017). Recientes estudios, como los de Aberg et al. (2006) o Mellor et al. (2017) consideran que la satisfacción corporal tiene un importante papel en el proceso de la satisfacción vital, ya que mantiene importantes conexiones con la autoestima, el estado anímico, la identidad de género y el autoconcepto personal. No obstante, la mayoría de los estudios que apoyan estas afirmaciones sobre las conexiones entre satisfacción corporal y vital se basan en muestras de personas jóvenes y de mediana edad (Frederick, Buchanan, Sadehgi-Azar, Peplau, Haselton, Berezovskaya, y Lipinski, 2006; González, 2017), por lo que el mayor interés de esta investigación es corroborar si la satisfacción corporal y vital siguen relacionándose en personas en proceso de madurez o vejez, ya que, de la fortaleza de esta asociación depende que la satisfacción corporal sea un tema de

$\begin{array}{llllllll}\text { Eur. J. } & \text { Health. } & \text { Research. } & \text { Vol. } & 4, & N^{\circ} & 2 & \text { (Págs. }\end{array}$ 
relevancia científica para las personas en proceso de madurez, o sea un aspecto más trivial.

Algunos estudios (Mangweth-Matzek et al., 2006; Vega et al., 2015) indican que la relación de la satisfacción corporal sobre la satisfacción vital podría mantenerse alta durante la madurez y la vejez, ya que la preocupación por la apariencia física no se reduce significativamente según se avanza en edad, e incluso se mantiene significativamente alta en personas de edad avanzada. En estos estudios se refleja cómo el sexo, la red social y la presencia de pareja tienen gran influencia sobre la satisfacción corporal y vital. Es de sumo interés valorar hasta qué punto esta relación de satisfacción corporal sobre satisfacción vital sigue siendo relevante para la persona con el paso de los años, valorar igualmente la influencia de factores asociados como el sexo, el hábitat y la presencia de pareja, y valorar también la posible existencia de un momento vital en el que ambos constructos dejen de relacionarse significativamente, ya que actualmente no existen estudios que aclaren estas cuestiones.

Buscando respuesta y confirmación a estas cuestiones, esta investigación se plantea dos objetivos: (1) Describir el grado de satisfacción corporal y vital de las personas mayores de 50 años sin problemas graves de salud en España, y (2) valorar las relaciones existentes entre ambas variables, teniendo en cuenta el sexo, edad, hábitat y la presencia o ausencia de pareja, como factores de gran relevancia para la satisfacción corporal y vital. La respuesta a estas dos cuestiones debe servir para certificar la importancia real de la satisfacción corporal durante la vejez, con vistas a mejorar la intervención sobre las personas mayores con estado de ánimo deprimido y baja autoestima, problemas que llegan a afectar a un 30-50\% de la población mayor (Yanguas, 2006).

\section{MÉTODO}

\section{Participantes}

Se ha utilizado 176 personas mayores de 50 años $(M=64.03$ años; $D T=8.06)$. 83 menores de 65 años y 93 mayores de 65 años. La distribución entre sexos es de 30 varones y 146 mujeres. La muestra ha sido extraída de la provincia de Zamora, por ser la provincia que mejor representa las estimaciones futuras de evolución de la población española, al presentar un índice de envejecimiento que duplica ampliamente la media nacional, siendo el segundo mayor índice de envejecimiento del estado español, sólo por detrás de Orense; a la que superará en 2019 según las estimaciones de la evolución de la población española (Abellán, Ayala, y Pujol, 2017). Se seleccionó a través de la participación en distintos programas sociales de Cruz Roja Española en Zamora, utilizándose para la obtención de los participantes un muestreo por 
conglomerados en las localidades elegidas. La distribución de los sujetos a las condiciones experimentales ha sido mediante muestreo aleatorio.

\section{Instrumentos}

Body Shape Questionnaire (BSQ. Cooper, Taylor, Cooper, y Fairburn, 1987): evalúa la satisfacción corporal y ha sido adaptado y validado sobre población española por Raich et al. (1996). Tiene una elevada fiabilidad interna ( $\alpha$ de Cronbach entre 0.95 y 0.97) y validez concurrente con otros instrumentos similares como el Multidimensional Body Self-Relations Questionnaire (MBSRQ. Cash, 2015) y la Escala de insatisfacción corporal del Eating Disorders Inventory (EDI. Garner, Olmstead, y Polivy, 1983). Consta de 34 ítems. Este instrumento ha sido seleccionado por su sencillez de aplicación a población envejecida, ya que tan sólo consta de 34 ítems y presenta un léxico sencillo (Baile et al., 2002).

Cuestionario de Satisfacción Vital (CSV. Sánchez, 2012). Valora la satisfacción vital a través de 15 ítems en una escala tipo Likert, y con un $\alpha$ de Cronbach .746. Este cuestionario también ha sido diseñado específicamente para cubrir la ausencia de instrumentos sobre satisfacción vital para personas mayores de bajo nivel cultural y sin experiencia en el ejercicio de autoevaluación. Las puntuaciones de los instrumentos se interpretan de forma inversa, a mayor puntuación menor satisfacción corporal y vital, y viceversa.

\section{Procedimiento}

Se ha realizado un estudio descriptivo de corte transversal de la satisfacción corporal y vital de las personas mayores de 50 años en la provincia de Zamora, valorando la influencia e interacción de diversos factores como sexo, edad (como variable dicotómica con punto de corte en los 65 años), hábitat (urbano-rural) y presencia o ausencia de pareja actual. Se obtuvo consentimiento informado de la muestra para el estudio.

Para la extracción de las inferencias estadísticas se ha realizado un Análisis Factorial de la Varianza considerando la influencia de los factores expuestos y sus interacciones simples.

Para la interacción de los factores combinados, al considerarse más de dos categorías, se utiliza el Análisis de las Comparaciones Múltiples Post Hoc, con el objetivo de especificar la localización de las diferencias significativas en alguna o algunas de las categorías resultantes. Para este análisis se ha utilizado la Prueba de Tukey, ya que las pruebas de homogeneidad no dieron resultados estadísticamente significativos $(\alpha=0.05)$ en ninguno de los casos.

Por último se utiliza el Coeficiente de Pearson como estadístico de contraste para el análisis correlacional entre variables e instrumentos.

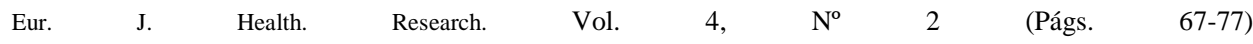




\section{RESULTADOS}

En respuesta al primer objetivo de la investigación, se describe en la tabla 1 el grado de satisfacción corporal y vital de las personas mayores de 50 años.

Tabla 1. Medias y desviaciones típicas de los instrumentos de evaluación según las variables consideradas

\begin{tabular}{|c|c|c|c|c|c|c|}
\hline & & \multicolumn{3}{|c|}{ BSQ } & \multicolumn{2}{|c|}{ CSV } \\
\hline & & $N$ & $M$ & $D T$ & $M$ & $D T$ \\
\hline \multirow{2}{*}{ SEXO } & Varón & 30 & 62.80 & 22.91 & 18.27 & 7.45 \\
\hline & Mujer & 146 & 72.13 & 23.30 & 21.49 & 6.28 \\
\hline \multirow{2}{*}{ EDAD } & 65 o mayor & 93 & 65.02 & 20.89 & 20.88 & 7.43 \\
\hline & Menor 65 & 83 & 76.72 & 24.69 & 21.00 & 7.30 \\
\hline \multirow{2}{*}{ ENTORNO } & Rural & 63 & 68.08 & 19.44 & 20.60 & 6.86 \\
\hline & Urbano & 113 & 71.91 & 25.37 & 21.12 & 7.63 \\
\hline \multirow[t]{2}{*}{ PAREJA } & $\begin{array}{l}\text { Con pareja } \\
\text { actual }\end{array}$ & 117 & 72.51 & 23.49 & 20.62 & 7.32 \\
\hline & Sin pareja actual & 59 & 66.62 & 23.03 & 21.58 & 7.42 \\
\hline Total & & 176 & 70.54 & 23.44 & 20.94 & 7.35 \\
\hline
\end{tabular}

En relación al factor sexo, se puede observar cómo las puntuaciones en Body Shape Questionnaire (BSQ) y en el Cuestionario de Satisfacción Vital (CSV) son más altas en mujeres que en varones. El ANOVA de un Factor realizado teniendo en cuenta esta variable, tanto en BSQ $(F=4.011$; Sig.=0.047) como en Cuestionario de Satisfacción Vital $(F=4.887$; Sig.=0.028), refleja que estas diferencias son estadísticamente significativas. Se puede concluir, por tanto, que los varones tienen mayor satisfacción corporal y vital.

En relación al factor edad, las personas menores de 65 muestran menor satisfacción corporal medida a través del BSQ. El ANOVA de un Factor realizado teniendo en cuenta esta variable en BSQ $(F=11.593$; Sig. $=0.001)$ refleja que estas diferencias observadas son estadísticamente significativas. Sin embargo, en satisfacción vital, medida a través del CSV, los resultados no son estadísticamente significativos.

El hábitat, urbano o rural, y la presencia o ausencia de pareja, no ofrecen resultados estadísticamente significativos según el ANOVA de un Factor en ninguna de las variables estudiadas.

Respecto al segundo objetivo del estudio, se muestran en la tabla 2 las correlaciones entre los instrumentos de medida y en la tabla 3 las relaciones existentes entre ambas variables teniendo en cuenta la interacción de las variables sexo, edad, hábitat y presencia o ausencia de pareja.

Tabla 2. Correlaciones entre los instrumentos de medida 


\begin{tabular}{ccc}
\hline & BSQ & CU.SV \\
\hline BSQ & 1 & 0.246 \\
\hline CU.SV & 0.246 & 1 \\
\hline
\end{tabular}

Todas las correlaciones observadas entre instrumentos son positivas y tienen una significación mayor al 99\% $(\alpha=0.01)$, lo que refleja que, tanto en relación a los instrumentos de medida, como a las variables estudiadas (satisfacción corporal y vital), existe una relación directa estadísticamente significativa que hace covariar significativamente satisfacción corporal y vital.

Tabla 3. Medias y desviaciones típicas de la interacción de las variables evaluadas

\begin{tabular}{|c|c|c|c|c|c|c|}
\hline & & & \multicolumn{2}{|c|}{ BSQ } & \multicolumn{2}{|c|}{ CSV } \\
\hline & & $N$ & $M$ & $D T$ & $M$ & $D T$ \\
\hline \multirow{4}{*}{ SEXO/EDAD } & Varón/ Menor 65 & 9 & 78.67 & 16.28 & 17.56 & 7.95 \\
\hline & Varón/Mayor 65 & 21 & 56.00 & 22.21 & 18.57 & 5.62 \\
\hline & Mujer/Menor 65 & 74 & 76.49 & 25.59 & 21.42 & 7.16 \\
\hline & Mujer/Mayor65 & 72 & 67.65 & 19.89 & 21.56 & 7.78 \\
\hline \multirow{4}{*}{ SEXO/HÁBITAT } & Varón/Rural & 11 & 67.91 & 25.09 & 17.64 & 4.78 \\
\hline & Varón/Urbano & 19 & 59.84 & 21.70 & 18.63 & 7.10 \\
\hline & Mujer/Rural & 52 & 68.12 & 18.33 & 21.23 & 7.10 \\
\hline & Mujer/Urbano & 94 & 74.35 & 25.47 & 21.63 & 7.67 \\
\hline \multirow{4}{*}{ EDAD/HÁBITAT } & Menor65/Rural & 23 & 74.52 & 16.38 & 22.78 & 6.05 \\
\hline & Menor65Urbano & 40 & 77.57 & 27.28 & 20.32 & 7.65 \\
\hline & Mayor65/Rural & 60 & 64.37 & 20.27 & 19.35 & 7.05 \\
\hline & Mayor65/Urbano & 53 & 65.51 & 21.53 & 22.04 & 7.57 \\
\hline \multirow{4}{*}{ SEXO/PAREJA } & Varón/Sin pareja & 9 & 62.78 & 19.82 & 16.44 & 6.35 \\
\hline & Varón/Con pareja & 21 & 62.80 & 24.58 & 19.05 & 6.24 \\
\hline & Mujer/Sin pareja & 50 & 67.32 & 23.67 & 22.50 & 7.27 \\
\hline & Mujer/Con pareja & 96 & 74.64 & 22.83 & 20.96 & 7.52 \\
\hline \multirow{4}{*}{ EDAD/PAREJA } & $<65 /$ Sin Pareja & 23 & 76.17 & 27.81 & 21.65 & 7.83 \\
\hline & $>65 /$ Con pareja & 60 & 76.93 & 23.63 & 20.75 & 7.13 \\
\hline & $<65 /$ Sin pareja & 36 & 60.53 & 17.16 & 21.53 & 7.26 \\
\hline & $>65 /$ Con pareja & 57 & 67.86 & 22.62 & 20.47 & 7.57 \\
\hline \multirow{4}{*}{$\begin{array}{l}\text { HÁBITAT/ } \\
\text { PAREJA }\end{array}$} & Rural/Sin pareja & 19 & 63.21 & 16.91 & 20.63 & 7.37 \\
\hline & Rural /Con pareja & 44 & 70.18 & 20.25 & 20.59 & 6.71 \\
\hline & Urba./Sin pareja & 40 & 68.25 & 25.46 & 22.06 & 7.50 \\
\hline & Urba./Con pareja & 73 & 73.92 & 25.27 & 20.63 & 7.70 \\
\hline Total & & 176 & 70.54 & 23.44 & 20.94 & 7.35 \\
\hline
\end{tabular}

La interacción de sexo y edad refleja diferencias estadísticamente significativas en satisfacción corporal medida con Body Shape Questionnaire $(F=5.383$; Sig.=0.001). Los varones mayores de 65 años muestran significativamente mayor satisfacción corporal respecto al resto de condiciones según el análisis Post Hoc realizado.

La interacción de hábitat y edad también refleja diferencias estadísticamente significativas en satisfacción corporal medida con BSQ $(F=3.945$; Sig.=0.009). Las personas menores de 65 años que habitan en entornos urbanos muestran significativamente menor satisfacción corporal.

Eur. J. Health. Research. Vol. 4, $\quad \mathrm{N}^{\mathrm{o}} 2$ (Págs. 
La interacción de edad y presencia o ausencia de pareja también refleja diferencias estadísticamente significativas en ambos instrumentos de satisfacción corporal $(F=4.642$; Sig. $=0.004)$. En el resto de interacciones simples no se observan diferencias estadísticamente significativas en este estudio.

\section{DISCUSIÓN Y CONCLUSIONES}

Debido a la ausencia de estudios españoles que evalúen la satisfacción corporal en personas mayores, y a la escasez de estudios que la evalúen en otros grupos de edades, no se cuenta con muchas referencias con las cuales establecer comparaciones de los resultados de este estudio. Cooper et al. (1987), en la validación original de Body Shape Questionnaire, reflejó una media en mujeres de origen anglosajón de 84.7 con una desviación típica de 28.4. La adaptación española de Raich et al. (1996a), mostró una puntuación media de 84.75 y una desviación típica de 30.42 entre estudiantes universitarias. Ya en el siglo XXI, Baile et al. (2002) con chicas adolescentes españolas, obtuvo media de 81.2 y desviación típica de 30.25 para chicas de 15-16 años y media de 79.49 y desviación típica de 31.61 para chicas de 17-19 años. Por último, el más reciente de Fernández et al. (2015), aunque no muestra medias y desviaciones típicas concretas, afirma haber obtenido resultados muy similares con su muestra de más de quinientas adolescentes y preadolescentes. Si se comparan los resultados obtenidos en este estudio sobre personas mayores de 50 años, con media de 70.54 y desviación típica 23.44 , se puede observar cómo, aunque existen diferencias claras entre los distintos grupos, los rangos que incluyen el $68 \%$ de los datos (rango que agrupa el área en torno a una desviación típica sobre la media) coinciden en más del $60 \%$ de los casos. Se puede concluir, por tanto, que gran parte de la población mayor de 50 años tiene niveles similares de insatisfacción corporal que grupos de adolescentes y jóvenes (Karazsia, Murnen, y Tylka, 2017).

La principal consecuencia de esta conclusión es que se reduce la influencia de la variable edad como generadora de cambios sobre la satisfacción corporal, ya que los diferentes grupos de edad muestran resultados similares, haciendo necesario valorar cuáles son aquellos factores que se combinan con el aumento de edad a partir de los 50 años para provocar una reducción significativa en la satisfacción corporal en algunas personas. Los resultados obtenidos con las interacciones simples de los factores estudiados apoyan esta afirmación, ya que son las interacciones combinadas de la variable edad con sexo, hábitat y presencia de pareja las que muestran diferencias estadísticamente significativas, es decir, las personas mayores de 65 años disminuyen significativamente su satisfacción corporal especialmente si son mujeres, de hábitats urbanos o tienen pareja actual.

Estos resultados apoyan la afirmación realizada por Kozar (2005) en su estudio, en el que concluyó que la edad no era el factor determinante para que se 
produzca una reducción considerable en la preocupación por el aspecto físico al llegar a la madurez y la vejez. Según Kozar, para que una persona reduzca considerablemente la preocupación por la apariencia física debe observar una gran diferencia con el ideal físico que se ha marcado, percibir un paulatino distanciamiento con ese ideal y considerar la imposibilidad de recortar la distancia. Kozar afirma que la alta covariación del factor edad con estos requisitos suele provocar la habitual confusión de las investigaciones que relacionan edad e insatisfacción corporal.

En relación al segundo objetivo de la investigación existe una correlación directa significativa entre satisfacción corporal y vital, tal y cómo predicen estudios previos (Vega et al., 2015; Zeman y Zeman, 2015). Aunque los resultados en los análisis post hoc reflejan que diferencias significativas en satisfacción corporal, no conllevan diferencias significativas en satisfacción vital en ninguna condición y variable considerada, salvo en la variable sexo. La explicación más probable de estos resultados es que la única variable de las consideradas en este estudio que influye sobre la satisfacción corporal y vital de forma similar que en la juventud es el sexo, tal y cómo postulan Davison y McCabe (2005) en su estudio longitudinal, en el que apreciaron cómo la imagen corporal y el funcionamiento social, psicológico y sexual funcionaban de forma similar en todas las edades con pequeñas excepciones. Según Davidson y McCabe la satisfacción corporal de los varones se asocia más con factores afectivos y sexuales, y en mujeres se asocia más con factores sociales y de interacción entre iguales a lo largo de toda la vida. Los resultados conjuntos en Body Shape Questionnaire y Cuestionario de Satisfacción Corporal en la interacción de sexo-presencia de pareja apoyan esta afirmación, ya que la mayor satisfacción corporal se da en varones con pareja y en mujeres sin pareja. Según Davison y McCabe, los varones tendrían cubiertas sus necesidades sexuales y afectivas al tener pareja estable, y las mujeres sin pareja estable mantendrían cubiertas sus necesidades sociales y de interacción entre iguales en mayor medida que mujeres con pareja estable, por lo que, tanto mujeres como varones, tenderían a despreocuparse más por su aspecto físico. Es decir, las diferencias observadas entre hombres y mujeres están camufladas a través del factor presencia de pareja, que tiene un funcionamiento inverso según el sexo, y por ello la correlación entre satisfacción corporal y vital parece funcionar de forma similar que durante la juventud. Se puede considerar, por lo tanto, que, en el caso de las mujeres, tener en cuenta la satisfacción corporal a la hora de plantear una intervención sobre un estado de ánimo depresivo sigue teniendo gran relevancia.

Finalmente, como conclusión general, se puede considerar que los resultados de este estudio reflejan como en el ámbito de la satisfacción corporal y vital en mayores es necesario un análisis en profundidad de los diversos factores implicados, ya que la no inclusión de alguno de ellos suele derivar en conclusiones erróneas o en diferencias significativas que pasan desapercibidas en análisis más globales, tal y como ocurre con 
el factor edad, que provoca diferencias significativas en satisfacción corporal combinado con los factores sexo, hábitat y presencia de pareja, o en la correlación entre satisfacción corporal y vital en el que las diferencias en el factor sexo se camuflan a través del factor presencia de pareja.

\section{REFERENCIAS}

Abellán, A., Ayala, A. y Pujol, R. (2017). Un perfil de las personas mayores en España, 2017. Indicadores estadísticos básicos. Informes Envejecimiento en red, 15, 41-48.

Aberg, A.C., Sindeval, B., Hepworth, M., O'Reilly, K. y Lithell, H. (2005). On loss of activity and independence, adaptation improves life satisfaction in old age. A qualitative study of patients' perceptions Quality of Life Research. An International Journal of Quality of Life Aspects of Treatment, Care and Rehabilitation. 14(4), 1111-1125.

Baile, J.I., Guillén, F. y Garrido, E. (2002). Insatisfacción corporal en adolescentes medida con el Body Shape Questionnaire (BSQ): Efecto del anonimato, el sexo y la edad. Revista Internacional de Psicología Clínica y de la Salud. 2(3), 439-450.

Boyes, A.D., Fletcher, G.J.O. y Latner, J.D. (2007). Male and female body image and dieting in the context of intimate relationships. Journal of Family Psychology, 21(4), 764-768.

Cash, T.F. (2015). Multidimensional Body-Self Relations Questionnaire (MBSRQ). In Encyclopedia of Feeding and Eating Disorders (pp. 1-4). Springer: Singapore.

Cash, T.F. (2017). Body Image: A joyous journey. Body Image, 23. A1-A2.

Castellano, C.L. (2014). La influencia del apoyo social en el estado emocional y las actitudes hacia la vejez y el envejecimiento en una muestra de ancianos. International Journal of psychology and psychological therapy, 14(3), 365-377.

Chacón-Borrego, F., Corral-Pernía, J.A., y Castañeda-Vázquez, C. (2017). Relación entre actividad física, estados de ánimo y género en personas adultas. European Journal of Health Research, 3(3), 163-171.

Cooper, P.J., Taylor, M.J., Cooper, Z. y Fairburn, C.G. (1987). The development and validation of the Body Shape Questionnaire. International Journal of Eating Disorders, 6, 485-494.

Davison, T.E., y McCabe, M.P. (2005). Relationships between men's and women's body image and their psychological, social, and sexual functioning. Sex Roles, 52(7-8), 463-475.

Frederick, D.A., Buchanan, G.M., Sadehgi-Azar, L., Peplau, L.A, Haselton, M.G., Berezovskaya, A. y Lipinski, R.E. (2007). Desiring the muscular ideal: Men's body satisfaction in the United States, Ukraine, and Ghana. Psychology of Men \& Masculinity, 8(2), 103-117.

Garner, D.M., Olmstead, M.P., y Polivy, J. (1983). Development and validation of a multidimensional eating disorder inventory for anorexia nervosa and bulimia. International journal of eating disorders, 2(2), 15-34.

González, A. (2017). ¿Cómo me veo?: Mujeres mayores hablan de los cambios corporales sufridos con el paso del tiempo. Revista Costarricense de Trabajo Social, (22), 29-40.

Gubrium, J.F., y Holstein, J.A. (2006). The Life Course. Handbook of symbolic interactionism. Walnut Creek, CA: AltaMira Press.

Homan, K.J., y Tylka, T.L. (2018). Development and exploration of the gratitude model of body appreciation in women. Body image, 25, 14-22.

Hudson, N.W., Lucas, R.E. y Donnellan, M.B. (2016). Getting older, feeling less? A crosssectional and longitudinal investigation of developmental patterns in experiential wellbeing. Psychology and Aging, 31(8), 847-861. 
Karazsia, B.T., Murnen, S.K. y Tylka, T.L. (2017). Is body dissatisfaction changing across time? A cross-temporal meta-analysis. Psychological bulletin, 143(3), 293-320.

Kozar, J.M. (2005). Relationship of attitudes toward advertising images and self-perceptions of older women. Dissertation Abstracts International Section A: Humanities and Social Sciences, 65(8), 3116.

Maganto, C., Garaigordobil, M. y Kortabarria, L., (2017). Eating Problems in Adolescents and Youths: Explanatory Variables. The Spanish Journal of Psychology, 19(81), 1-9. doi.10.1017/sjp.2016.74

Mangweth-Matzek, B., Rupp, C.I., Hausmann, A., Assmayr, K., Mariacher, E., Kemmler, G., ... y Biebl, W. (2006). Never too old for eating disorders or body dissatisfaction: A community study of elderly women. International Journal of Eating Disorders, 39(7), 583-586.

Markey, C.N., Markey, P.M. y Birch, L.L. (2001). Interpersonal predictors of dieting practices among married couples. Journal of Family Psychology, 15, 464-475.

Mellor, D., Connaughton, C., McCabe, M.P. y Tatangelo, G. (2017). Better with age: A health promotion program for men at midlife. Psychology of Men \& Masculinity, 18(1), 40-49.

Murray, T., y Lewis, V. (2014). Gender-role conflict and men's body satisfaction: The moderating role of age. Psychology of Men \& Masculinity, 15(1), 40-48.

Parra-Rizo, M.A. (2017). Componentes de influencia más valorados en la calidad de vida por las personas mayores de 60 años físicamente activas. European Journal of Investigation in Health, Psychology and Education, 7(3), 135-144. doi: 10.30552/ejihpe.v7i3.202

Raich, R.M. (2004). Una perspectiva desde la Psicología de la Salud de la Imagen Corporal. En Revista Latinoamericana de Psicología. Número monográfico: Psicología de la Salud de la Mujer. Avances en Psicología Latinoamericana, 22, 15-27.

Raich, R.M., Mora, M., Soler, A., Ávila, C., Clos, I. y Zapater, L. (1996). Adaptación de un instrumento de evaluación de la insatisfacción corporal (BSQ). Clínica y Salud, 8, 5166.

Rosen, J.C. (2006). Eating Disorders: Pulling it all Together. PsycCRITIQUES, ElectronicCollection.

Sabik, N., y Versey, H.S. (2016). Functional limitations, body perceptions, and health outcomes among older African American women. Cultural Diversity and Ethnic Minority Psychology, 22(4), 594-601.

Sánchez, R. (2012). IMAGINA, Programa de Mejora de la Autoestima y la Imagen Corporal para Adultos. Logroño: Editorial Sinindice.

Sánchez, R. y Maganto, C. (2018). Body Image and Old Age. Descriptive Study of the Spanish Social Reality. Journal of Education and Human Development, 7(1), 25-36. Recuperado de: http://jehdnet.com/journals/jehd/Vol_7_No_1_March_2018/4.pdf

Thompson, J.K. (2004). Handbook of Eating Disorders and Obesity. Hoboken, NJ: Wiley.

Yanguas, J.J. (2006). Análisis de la calidad de vida relacionada con la salud en la vejez desde una perspectiva multidimensional. Colección estudios: Personas Mayores. Madrid: IMSERSO.

Zeman, Z., y Zeman, M.G. (2015). "Young" heart in "old" body. Experiences of ageing from older persons' perspective. Etnološka tribina, 38 .

Recibido: 06 de julio de 2018

Recepción Modificaciones: 18 de julio de 2018

Aceptado: 24 de julio de 2018

Eur. J. Health. Research. Vol. 4, $\mathrm{N}^{\mathrm{o}} 2$ (Págs. 
SÁNCHEZ, BARRIENTOS y ARIGITA. La madurez ante el espejo 